\title{
CLUSTER SETS OF PSEUDO- MEROMORPHIC FUNCTIONS
}

\author{
D. A. STORVICK ${ }^{11}$
}

Let $w=f(z)=u(x, y)+i v(x, y)$ be an interior transformation in the sense of Stoilow ${ }^{2)}$ in an arbitrary domain $D$, i.e. $w=f(z)$ is continuous and singlevalued in $D$, and unless constant takes open sets in $D$ into open sets in the $w$-plane, and does not take any continuum (other than a single point) into a single point of the $w$-plane. Stoilow proved that such an interior transformation can be represented as

$$
f(z)=\varphi(T(z))
$$

where $T(z)=\xi$ is a topological mapping of $D$ onto a domain $D^{\prime}$ and $w=\varphi(\xi)$ is a meromorphic function in $D^{\prime}$. If the homeomorphism $\xi=T(z)$ is a quasiconformal mapping ${ }^{3)}$ we shall call $w=f(z)$ a pseudo-meromorphic function and if in addition $w=\varphi(\xi)$ is analytic, we shall term $w=f(z)$ pseudo-analytic. (A point $z_{0} \in D$ is a pole of order $n$ of $w=f(z)$ if $\varphi(\xi)$ has a pole of order $n$ at $\left.\xi_{0}=T\left(z_{0}\right)\right)$; the definition of essential singularity for a pseudo-meromorphic function is given in an analogous fashion.

We shall restrict our attention to functions $w=f(z)$ defined and pseudomeromorphic in $|z|<1$. We make no assumptions about the unit circle $|z|=1$, on which some of the points may be regular points, poles or essential singularities.

The cluster set of $f(z)$ at $z_{0},\left|z_{0}\right|=1, C\left(f, z_{0}\right)$, is the set of all points $\alpha$ for which there exists a sequence $\left\{z_{n}\right\},\left|z_{n}\right|<1$, such that $\lim _{n \rightarrow \infty} z_{n}=z_{0}$ and $\lim _{n \rightarrow \infty} f\left(z_{n}\right)=\alpha$.

Received August 3, 1960.

1) This paper was written under Contract No. AF $49(638)-836$ with the Air Force Office of Scientific Research.

2) See Stoilow [10]. The numbers in brackets refer to the bibliography.

3) A one to one function $w=f(z)=u(x, y)+i v(x, y)$ of class $C^{1}$ and positive Jacobian in a domain $\Omega$ is called quasi-conformal in $\Omega$ if there exists a constant $M \geq 1$ such that $E+G \leq 2 M \vee E G-F^{2}$ in $\Omega$, where $E=u_{x}{ }^{2}+v_{x}{ }^{2}, F=u_{x} u_{y}+v_{x} v_{y}$ and $G=u_{y}{ }^{2}+v_{y}{ }^{2}$. See Mori [6] for relations between this and other common definitions of quasi-conformal mappings. 
Caratheodory [2] established for functions bounded and analytic in $|z|<1$ an important theorem relating the cluster set at a point to the radial limit values at neighboring points. His techniques can be modified so as to yield the same result for pseudo-analytic functions. First a lemma will be proved.

Lемма. Let $w=f(z)$ be a bounded pseudo-analytic function in $|z|<1$, $|f(z)|<M$. Let $W_{z}$ denote the set of radial limit values, $W_{2}=\{w \mid w=$ $\lim _{\rightarrow \rightarrow 1} f\left(r e^{i \theta}\right)=f^{*}\left(e^{i \theta}\right), e^{i \theta}$ on $\left.|z|=1\right\}$. Then $f(0)$ is contained in the closure of the convex hull of $W_{z}, f(0) \in \overline{\mathrm{C} . \mathrm{H} .}\left\{W_{z}\right\}$.

Proof: Because the Riemann surface $\Re$ of the inverse function $f^{-1}(w)=z$ is of hyperbolic type, let $\xi=\varphi^{-1}(w)$ be the function which maps $\mathfrak{R}$ one-to-one conformally onto $|\xi|<1$. The function $w=\varphi(\xi)$ is analytic in $|\xi|<1$. Setting $\xi=\varphi^{-1}(w)=\varphi^{-1}(f(z))=T(z)$ we see that $\xi=T(z)$ is a univalent pseudo-analytic function giving a quasi-conformal mapping of $|z|<1$ onto $|\xi|<1$. The function $w=\varphi(\xi)$ can be chosen so that $\varphi(0)=f(0)$ and hence $T(0)=0$.

Since $w=\varphi(\xi)$ is a bounded analytic function in $|\xi|<1$, there exist radial limit values $\lim _{r \rightarrow 1} \varphi\left(r e^{i \theta}\right)=\varphi^{*}\left(\mathrm{e}^{i \theta}\right)$ for almost all $e^{i \theta}$. Let $W_{\xi}=\left\{w \mid w=\varphi^{*}\left(e^{i \theta}\right), e^{i \theta}\right.$ on $|\xi|=1\}$. From the well-known result of Carathéodory, $\varphi(0) \in \bar{C} . \mathbf{H} .\left\{W_{\xi}\right\}$ and hence the result will follow after proving $W_{\xi} \subseteq W_{z}$. (Actually we'll prove $W_{\xi}=W_{z}$; this is interesting because the set of points on $|\xi|=1$ for which the radial limit values of $\varphi(\xi)$ fail to exist is of measure zero while the set of points on $|z|=1$ for which the radial limit values of $f(z)$ fail to exist may be of positive measure).

Let $f^{*}\left(e^{i \theta_{0}}\right) \in W_{z}$ and let $e^{i \beta}=T\left(e^{i \theta_{0}}\right)$, (the quasi-conformal mapping $\xi=T(z)$ can be extended to give a homeomorphism between $|z| \leq 1$ and $|\xi| \leq 1$ ). The radius $R_{\theta=\theta_{0}}$ in $|z|<1$ is mapped by $\xi=T(z)$ onto a Jordan $\operatorname{arc} L$ lying in $|\xi|<1$ and terminating at $e^{i \beta}$ on $|\xi|=1$. Since $w=\varphi(\xi)=f\left(T^{-1}(\xi)\right)$ it follows that there exists the limit of $\varphi(\xi)$ as $\xi \rightarrow e^{i \beta}$ along $L$. By Lindelöf's theorem $[7 ;$ p. 70$]$ therefore, there exists the radial $\operatorname{limit} \lim _{r \rightarrow 1} \varphi\left(r e^{i \beta}\right)=\varphi^{*}\left(e^{i \beta}\right)=f^{*}\left(e^{i 0_{0}}\right)$. Therefore $f^{*}\left(e^{i \theta_{0}}\right) \in W_{\text {氵 }}$ and $W_{z} \subseteq W_{\xi}$.

To prove $W_{\xi} \subseteq W_{z}$, let $\varphi^{*}\left(e^{i \gamma}\right) \in W_{\xi}$. The radius $R_{\theta=r}$ in $|\xi|<1$ maps onto a Jordan arc $\lambda$ in $|z|<1$ terminating at $e^{i \delta}$. From $w=f(z)=\varphi(T(z))$ it follows that the limit of $f(z)$ as $z \rightarrow e^{i \delta}$ along $\lambda$ exists and equals $\varphi^{*}\left(e^{i \Upsilon}\right)$. From a recent extension of Lindelöf's theorem $[11 ;$ p. 136$]$ it follows as above that $f^{*}\left(e^{i \delta}\right)$ 
$=\varphi^{*}\left(e^{i r}\right)$ and so $W_{\xi}=W_{z}$ and the lemma is proved.

It might be appropriate to point out at this time that this result cannot be extended to interior transformations, even though they are one-to-one, without some bound on local distortion.

The transformation $T_{1}$ given by

$$
\left\{\begin{array}{l}
\varphi(x, y)=\left[\frac{-1}{6\left|y-\frac{1}{2}\right|-4}\right] x+\left[\frac{6\left|y-\frac{1}{2}\right|-3}{6\left|y-\frac{1}{2}\right|-4}\right] \\
\varphi(x, y)=y
\end{array}\right.
$$

maps the interior of the pentagon with vertices at $(0,0),(1,0),(1,1),(0,1)$ and $\left(-3, \frac{1}{2}\right)$ homeomorphically onto the square with vertices at $(0,0),(1,0)$, $(1,1),(0,1)$ and the point $\left(-1, \frac{1}{2}\right)$ onto the point $\left(\frac{1}{2}, \frac{1}{2}\right)$. The transformation $T_{2}$ given by $\left\{\begin{array}{l}\phi(x, y)=x \\ \psi(x, y)=x y\end{array}\right.$ maps the interior of this square onto the interior of the triangle with vertices at $(0,0),(1,0)$ and $(1,1)$ and the point $\left(\frac{1}{2}, \frac{1}{2}\right)$ onto the $\left(\frac{1}{2}, \frac{1}{4}\right)$. The transformation $T_{3}$ given by an appropriate conformal mapping (Schwarz-Christoffel transformation followed by linear fractional transformation if it is desired explicitly) maps the interior of this triangle onto the interior of the unit circle and the point $\left(\frac{1}{2}, \frac{1}{4}\right)$ onto the origin and the origin on the point $(1,0)$.

The transformation $w=f(z)$ given by $f(z)=T_{1}^{-1}\left(T_{2}^{-1}\left(T_{3}^{-1}(z)\right)\right)$ sends $|z|<1$ homeomorphically onto the interior of the pentagon, $f(0)=-1+\frac{i}{2} \cdots$. Except for $e^{i \theta}=1$, the radial limits of $f(z)$ exist and lie on the lines joining $(0,0)$ to $(1,0),(1,0)$ to $(1,1)$ and $(1,1)$ to $(0,1)$. Clearly $f(0)$ does not belong to the closure of the convex hull of the radial limit values.

For a bounded pseudo-analytic function in $|z|<1, w=f(z)=\varphi(T(z))$, a point $\alpha \in C\left(f, e^{i \theta}\right)$ if and only if $\alpha \in C\left(\varphi, T\left(e^{i \theta}\right)\right)$. The set of radial limit values of $f(z)$ on an $\operatorname{arc} A$ of $|z|=1$ equals the set of radial limit values of $\varphi(\xi)$ on $T(A)$ since the two limits

$\lim _{r \rightarrow 1} f\left(r e^{i \theta}\right)$ and $\lim _{r \rightarrow 1} \varphi\left(r T\left(e^{i \theta}\right)\right)$ either simultaneously exist and are equal, or both fail to exist. By the technique above, Carathéodory's theorem can thus be extended to pseudo-analytic functions in the following form: 
THEOREM: If $w=f(z)$ is a bounded pseudo-analytic function in $|z|<1$, then the cluster set of $f(z)$ at $e^{i 0_{0}}, C\left(f, e^{i \theta_{0}}\right)$, is contained in the closure of convex hull of the radial limit values ${ }^{4)}$ (where they exist) on any arc $A=\left\{e^{i \theta} \mid \theta_{1}<\theta<\theta_{2}\right\}$ of which $e^{i \theta_{0}}$ is an interior point,

$$
C\left(f, e^{i \theta_{0}}\right) \subseteq \overline{\mathrm{C}} \overline{\mathrm{H}} .\left\{W_{z}(A)\right\} .
$$

Certain subsets of the cluster set $C\left(f, e^{i \theta}\right)$ will be of value to us in describing the behaviour of a function near the boundary of its domain of definition. The boundary cluster set $C_{B}\left(f, e^{i 0_{0}}\right)$ of $f(z)$ at $e^{i \theta_{0}}$ is defined in this manner:

$$
C_{F}\left(f, e^{i 0_{0}}\right)=\bigcap_{\eta>0}\left\{\bigcup_{0<\left|\theta-\theta_{0}\right|<\eta} C\left(f, e^{i \theta}\right)\right\}
$$

For a set $E$ of logarithmic capacity zero on $|z|=1$, the boundary cluster set of $f(z)$, modulo $E$, at $e^{i \eta_{0}}$ is defined in this manner:

$$
C_{B-E}\left(f, e^{i \theta_{0}}\right)=\bigcap_{\eta_{>0}}\left\{\bigcup_{\substack{0<\left|\theta-\theta_{0}\right|<\eta \\ e^{i \theta \notin E}}} C\left(f, e^{i \theta}\right)\right\}
$$

The radial cluster set $C_{p}\left(f, e^{i \theta}\right)$ is that subset of $C\left(f, e^{i \theta}\right)$ obtained by requiring the sequence $\left\{z_{n}\right\}$ to lie on the radius to $e^{i \theta}$. The radial boundary cluster set of $f(z)$, modulo $E$, at $e^{i \theta_{0}}$ is defined as follows:

$$
C_{R-E}\left(f, e^{i \theta_{0}}\right)=\bigcap_{\eta>0}\left\{\bigcup_{\substack{0<\left|-\theta_{0}\right|<\eta \\ e^{i \theta \notin E}}} C_{\rho}\left(f, e^{i \theta}\right)\right\}
$$

It was stated in [8] that Ohtsuka had proved that every value of $C\left(f, e^{i \theta_{0}}\right)$ $-C_{B}\left(f, e^{i 0_{0}}\right)$ is assumed by a pseudo analytic function $f(z)$ infinitely often in any neighborhood of $e^{i \theta_{0}}$ except for a set of measure zero, extending a result of Kametani-Tsuji ([4], [12], and [13]). In a very interesting recent paper, On the theorems of Gross and Iversen, Lohwater [5] proved several results concerning the larger set $C\left(f, e^{i 0_{0}}\right)-C_{R-E}\left(f, e^{i 0_{0}}\right)$ for meromorphic $f(z)$. We also would like to call attention to the paper of Woolf [16] who announces some related results. We shall now prove two theorems which are modifications of Lohwater's results. In 1953 Noshiro [8] pointed out the need for extending the result of Kametani-Tsuji and his own results (see Theorem 6 of.

4) Recall that the radial limit many fail to exist on a subset of positive measure of the $\operatorname{arc} A$. 
$[8])^{*)}$.

THEOREM. If $w=f(z)$ is pseudo-meromorphic in $|z|<1$ and if $E$ is an arbitrary set of capacity zero on $|z|=1$, then every value of $C\left(f, e^{i \theta_{0}}\right)-C_{R-F}(f$, $\left.e^{i \theta_{0}}\right)$ is assumed by $f(z)$ in any neighborhood of $e^{i \theta_{0}}$ except possibly for a set of capacity zero.

Assume there exists a point $e^{i \theta_{0}}$ on $|z|=1$ and a closed set $\mathfrak{F} \subseteq C\left(f, e^{i \theta_{0}}\right)$ $-C_{R-E}\left(f, e^{i \theta_{0}}\right)$, cap $\mathfrak{F}>0$, such that no point of $\mathfrak{F}$ is assumed infinitely often in some neighborhood of $e^{i \theta_{0}}$. There is clearly no loss of generality in assuming that $w=0$ and $w=\infty$ are elements of $\widetilde{F}$ and the portion of $\mathfrak{F}$ lying in the circle $|w|<\delta$ has positive capacity for every $\delta>0$. Since neither 0 nor $\infty$ is assumed infinitely often in some neighborhood of $e^{i \theta_{0}}$ we can find $\rho_{0}$ such that $f(z)$ is different from 0 and $\infty$ in $V_{p_{0}}=\left\{z|| z-e^{i \theta_{0}} \mid<\rho_{0}\right\}$. We can further find $\eta_{0}>0$ and $\varepsilon_{0}>0$ such that $\liminf _{r \rightarrow 1}\left|f\left(r e^{i \theta}\right)\right|>\varepsilon_{0}>0$ for all $\theta$ in $0<\left|\theta-\theta_{0}\right|<\eta_{0}$ such that $e^{i \theta}$ does not belong to $E$. If not, there would exist a sequence $\left\{\theta_{k}\right\}$ such that $\lim _{k \rightarrow \infty} \theta_{k}=\theta_{0}$ and such that $\lim _{k \rightarrow \infty}\left\{\lim _{r \rightarrow 1} \inf \left|f\left(r e^{i \theta_{k}}\right)\right|\right\}=0$. This implies that

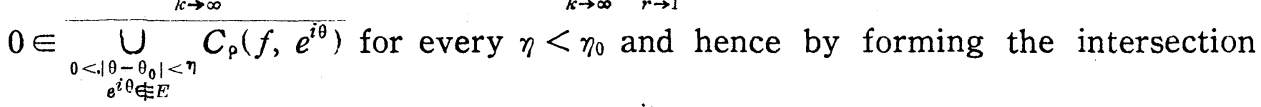
over all $\eta>0$ we see that $0 \in C_{R-F}\left(f, e^{i \theta_{0}}\right)$, and therefore 0 is not an element of $C\left(f, e^{i \theta_{0}}\right)-C_{R-E}\left(f, e^{i \theta_{0}}\right)$.

Consider the open set $H_{n}, H_{n}=\left\{z|| z|<1| f,(z) \mid<\frac{1}{n}\right\}$, for $\frac{1}{n}<\varepsilon_{0}$. We shall show that there exists a value of $n$ such that at least one component $G$ of $H_{n}$ is contained completely in $V_{p_{0}}$. Since $0 \in C\left(f, e^{i \theta_{0}}\right)$, there are points of $H_{n}$ in every neighborhood of $e^{i \theta_{0}}$, so that if no component of $H_{n}$ lies wholly in $V_{\rho_{0}}$, we can find a point in $V_{\rho_{0} / 2}$ and a point of $\left|z-e^{i \theta_{0}}\right|=\rho_{0}$ which are joined by a continuum $\Omega_{1}$ of the frontier of $H_{n+1}, \operatorname{Fr}\left(H_{n+1}\right)$, a point of $V_{\rho_{0} / z^{2}}$ and a point of $\left|z-e^{i \theta_{0}}\right|=\rho_{0}$ which are joined by a continuum $\AA_{2}$ of $\operatorname{Fr}\left(H_{n+2}\right)$ and, in general a point of $V_{\mathrm{P}_{0} / 2^{p}}$ and a point of $\left|z-e^{i \theta_{0}}\right|=\rho_{0}$ which are joined by a continuum $\Re_{p}$ of $F r\left(H_{n+p}\right)$. This bounded infinite sequence of continua $\left\{\Re_{p}\right\}$ must contain a subsequence $\left\{\Re_{p_{j}}\right\}$ which converges to a limiting continuum $\mathscr{N}_{0}$ joining

*) Added November 8, 1960. After this paper was submitted, the author learned of a related work by Noshiro, "Cluster sets of pseudo-analytic function", Japanese Journal of Mathematics 1959 (Vol. 29), pp. 83-91, March 1960 and also that the paper of Lohwater, "On the theorems of Gross and Iversen" has now appeared in volume VII of the Journal d'Analyse Mathematique and hence is more widely available. 
$e^{i \theta_{0}}$ to a point of $\left|z-e^{i \theta_{0}}\right|=\rho_{0}$. Since $\Re_{n+p}$ is a part of the level curve $|f(z)|$ $=\frac{1}{n+p}$, no point of $\Re_{0}$ can lie inside the unit circle since then $f(z)=0$ on a non-degenerate continuum which is impossible since $w=f(z)$ is an interior transformation. Therefore $\Omega_{0}$ is an arc of $|z|=1$ containing $e^{i \theta_{0}}$, and every radius of $|z|<1$ terminating at an interior point of $\Omega_{0}$ must cross infinitely many of the $\left\{\mathscr{R}_{p_{j}}\right\}$; so that $0 \in C\left(f, e^{i \theta}\right)$ for every $e^{i \theta} \in \Re_{0}$ which contradicts our assumption that $0 \in C\left(f, e^{i \theta_{0}}\right)-C_{R-E}\left(f, e^{i \theta_{0}}\right)$. Thus there exists at least one integer $n$ with $\frac{1}{n}<\varepsilon_{0}$ such that at least one component $G$ of $H_{n}$ lies wholly in $V_{\rho_{0}}$.

It is an immediate consequence of the maximum and minimum modulus principles for $f(z)$ that $G$ is simply connected and that the intersection $M=\bar{G}$ $\cap\{|z|=1\}$ is non-empty. We shall prove that the frontier of $G, \operatorname{Fr}(G)$, is locally connected. Now that part of $\operatorname{Fr}(G)$ lying inside $|z|<1$ consists of a piecewise smooth curve because it is the locus of points where $|f(z)|=\frac{1}{n}$. If $P$ is a point of $M$, and if $\operatorname{Fr}(G)$ is not locally connected at $P$, then, by a wellknown theorem [15; p. 19], there exists a non-degenerate subcontinuum $H$ of $\operatorname{Fr}(G)$ containing $P$ and such that $\operatorname{Fr}(G)$ is not locally connected at any point of $H$. Since $H$ must lie on $|z|=1$, it follows that $H$ is an arc of $|z|=1$. Moreover, there exists [15; p. 18] a circular neighborhood $V(P)$ of $P$ and a sequence of mutually disjoint components $N_{1}, N_{2}, N_{3}, \ldots$ of $F r(G) \cap V(P)$ converging to a nondegenerate limiting arc $N \subset H$ containing $P$. If $R$ is a circular neighborhood the midpoint of $N$, of radius less than one-fourth the length of $N$, it follows that a radius of $|z|<1$ drawn to any point $Q$ of that sub-arc $N_{0}$ cut out of $N$ by $R$ must cross the components $N_{j}$ of $F r(G) \cap V(P)$ arbitrarily close to $Q$. Along such a radius of $|z|<1$ we have $\lim _{r \rightarrow 1} \inf \left|f\left(r e^{i \theta}\right)\right|$ $\leq \frac{1}{n}<\varepsilon_{0}$, which contradicts the assumption that

$$
\lim _{r \rightarrow 1} \inf \left|f\left(r e^{i \theta}\right)\right|>\varepsilon_{0}
$$

for all $\theta$ in $0<\left|\theta-\theta_{0}\right|<\eta$ such that $e^{i \theta}$ does not belong to $E$.

To prove the set $M=\operatorname{Fr}(G) \cap\{|z|=1\}$ has logarithmic capacity zero we denote by $\widetilde{E}$ the complement of $E$ on $|z|=1$, and consider the decomposition of the set $M, M=(M \cap E) \cup(M \cap \widetilde{E})$. Since $E$ and therefore $M \cap E$ has capacity zero it will suffice to prove $M \cap \widetilde{E}$ has capacity zero. In $G,|f(z)|$ 
$<\frac{1}{n}$, so for $e^{i \theta} \in M, \lim _{z \rightarrow e i \theta} \sup |f(z)| \leq \frac{1}{n}$, while for $e^{i \theta} \in \widetilde{E}, \lim _{r \rightarrow 1} \inf \left|f\left(r e^{i \theta}\right)\right|>\varepsilon_{0}$ $>\frac{1}{n}$. Because $\operatorname{Fr}(G)$ is locally connected, each point is arcwise accessible from $G$, and along any arc of $G$ terminating at a point of $M$, the curvilinear cluster set ${ }^{6)}$ of $|f(z)|$ can contain no number greater than or equal to $\frac{1}{n}$. The radial cluster set of $\{f(z)\}$ at points of $\widetilde{E}$ can contain no point of modulus less than or equal to $\varepsilon_{0}$. By a result of Bagemihl ${ }^{7}, M \cap \widetilde{E}$ is at most denumerable and consequently has logarithmic capacity zero.

If the function $z=z(\xi)$ maps $|\xi|<1$ one-to-one conformally onto $G$, then the function the composition function $F(\xi)=n f(z(\xi))$ is a bounded pseudoanalytic function in $|\xi|<1,|F(\xi)|<1$. Since the image $M$ ₹ of $M$ under $z=z(\xi)$ has capacity zero [14 p. 347], the function $F(\xi)$ has radial limits of modulus 1 for all $e^{i \theta}$ except for a set of capacity zero. From a recent result of the author (see Theorem 6 of [11]) it must assume in $|\xi|<1$ all values in $|w|<1$ except possibly a set of capacity zero. That these values are assumed infinitely often follows from the factorization of $F(\xi)=\varphi(T(\xi))$ into a composition of a quasiconformal mapping of $|\xi|<1$ onto $|\zeta|<1$ and a bounded analytic function, $w=\varphi(\zeta),|\varphi(\zeta)|<1$. The function $w=\varphi(\zeta)$ has radial limits of modulus 1 for all $e^{i \theta}$ except for a set of capacity zero, and because it is neither constant nor a finite Blaschke product, by a theorem of Frostman [3] we can conclude it assumes infinitely often in $|\zeta|<1$ all values in $|w|<1$ except for a set of capacity zero and hence so does $F(\xi)$. Since $\rho_{0}$ may by taken arbitrarily small, the theorem is proved.

The relationship between exceptional values and asymptotic values is described in the following result.

THEOREM. If $w=f(z)$ is a pseudo-meromorphic function in $|z|<1$ and if $E$ is an arbitrary set of capacity zero on $|z|=1$ and if a value $\alpha$ of $C\left(f, e^{i \theta_{0}}\right)$ $-C_{R-E}\left(f, e^{i \theta_{0}}\right)$ is not assumed by $f(z)$ in some neighborhood of $e^{i \theta_{0}}$ then $\alpha$ is an asymptotic value of $f(z)$ either at $e^{i \theta_{0}}$ or else at a sequence of points having $e^{i \theta_{0}}$ as a limit point.

From the proof of the previous theorem it follows that we may assume

5) The subset of cluster set obtained by restricting the $z_{n}$ to lie on the arc.

6) See Theorem 2 of $[1 ;$ p. 380]. 
that the omitted value is $\alpha=0$, and that there exists a neighborhood $V_{p_{0}}$ of $e^{i n_{0}}$ such that $\liminf _{r \rightarrow 1}\left|f\left(r e^{i \theta}\right)\right|>\varepsilon_{0}>0$ for all $e^{i \theta}$ in $0<\left|\theta-\theta_{0}\right|<\eta$ such that $e^{i_{0}}$ does not belong to $E$. There exists an integer $n$ for which at least one component $G$ of the open set $H_{n}=\left\{z|| z|<1| f,(z) \mid<\frac{1}{n}<\varepsilon_{0}\right\}$ is completely contained in $V_{\rho_{0}}$.

For any $n$, the component $G$ must contain at least one component of $H_{n+1}$, for otherwise in $G$ the inequality $\frac{1}{n+1} \leq|f(z)|<\frac{1}{n}$ would be valid with $|f(z)|$ $=\frac{1}{n}$ at all points of $\operatorname{Fr}(G)$ except for a set $r \cap \lambda$ of capacity zero on the outer contour $r$ of $G$. Thus the extended maximum principle for pseudo-analytic functions [11, p. 133] is applicable and since $\frac{1}{f(z)} \leq n+1$ for all points of $G$ and $\lim \sup \left|\frac{1}{f(z)}\right| \leq n$ except for a set of capacity zero, we conclude that $\frac{1}{f(z)}<n$ in $G$ hence $|f(z)|>\frac{1}{n}$ which contradicts the structure of the region. Consequently we can construct a nested sequence of components $G=G_{0} \supset G_{1}$ $\supset G_{2} \supset G_{3} \supset \ldots \supset G_{k} \supset \ldots, G_{k} \subset H_{n+k}$. The intersection $\cap \overline{G_{k}}$ must consist of a single point on $|z|=1$; for, otherwise, this intersection must be a continum. If this continuum lies on $|z|=1$, we would then contradict our assumption that $\lim _{r \rightarrow 1} \inf \left|f\left(r e^{i \theta}\right)\right|>\varepsilon_{0}$ for all $e^{i \theta}$ with $0<\left|\theta-\theta_{0}\right|<\eta$ such that $e^{i \theta}$ does not belong to $E$. If part of this continuum lies in $|z|<1$, we contradict the assumption that $f(z) \neq 0$ in $V_{\rho_{0}}$.

We now choose a sequence of points $\left\{z_{k}\right\}, z_{k} \in G_{k}$ and form simple arc $l$ (or polygonal path) connecting $z_{1}$ with each $z_{k}$, lying in the nested sequence of components $G_{1} \supset G_{2} \supset \ldots \supset G_{k} \supset \ldots$ such that it terminates at the point $q=\cap G_{k}$ on $|z|=1$. Clearly $f(z) \rightarrow 0$ as $z \rightarrow q$ along $l$. Since $\rho_{0}$ may be taken arbitrarily small, we have 0 as an asymptotic value of $f(z)$ either at $e^{i \theta_{0}}$ or at a sequence of points on $|z|=1$ having $e^{i 0_{0}}$ as a limit point.

\section{BIBLIOGRAPHY}

[1] F. Bagemihl, Curvilinear cluster sets of arbitrary functions, Proc. Nat. Acad. Sci. (U.S.A.), 41 (1955), pp. 379-382.

[2] C. Carathéodory, Zum Schwarzschen Spiegelungsprinzip, Comm. Math. Helv., 19 (194647), 263-278.

[3] O. Frostman, Potential d'equilibre et capacité des ensembles, Thèse Lund, (1935).

[4] S. Kametani: The exceptional values of functions with the set of capacity zero of essential singularities, Proc. Acad. Tokyo, 17 (1941), pp. 429-433. 
[5] A. J. Lohwater, On the theorems of Gross and Iversen, Air Force Report AFOSR TN 59-550, AD 216660 (1959), pp. 1-16.

[6] A Mori, On quasi-conformality and pseudo-analyticity, Trans. Amer. Math. Soc., 84 (1957), pp. 56-77.

[7] R. Nevanlinna, Eindeutige analytische Funktionen, 2nd ed. Berlin (1953).

[8] K. Noshiro, On the theory of cluster sets of analytic functions, Sugaku, 5 (1953), pp. 65-72, AMS Translations, Series, 2, 8 (1958), pp. 1-12.

[ 9 ] A. Pfuger, Extremallängen und Kapazität, Comm. Math. Helv., 29 (1955), pp. 120-131.

[10] S. Stölcw, Leçons sur les principes topologiques de la théorie des fonctions analytiques, Paris (1938).

[11] D. A. Storvick, On pseudo-analytic functions, Nagcya Math. Journal, Nagoya Math. Journal, 12 (1957), pp. 131-138.

[12] M. Tsuji, On the cluster set of a meromorphic function, Proc. Acad. Tokyo, 19 (1943).

[13] M. Tsuji, On the Riemann surface of an inverse function of a meromorphic function in the neighborhood of a closed set of capacity zero, Proc. Acad. Tokyo, 19 (1943).

[14] M. Tsuji, Potential theory in modern function theory, Maruzen Co., Ltd., Tokyo, (1959).

[15] G. T. Whyburn, Analytic topology, Amer. Math. Soc. Colloq. Pub., 28(1942).

[16] W. B. Woolf. Radial cluster sets and the distribution of valucs of meromorphic functions, Notices Amer. Math. Scc., Abstract, 562-19, 6 (1959), p. 762.

Depariment of Mathematics

University of Minnesota 